Pacific Journal of Mathematics

ON THE PERIODICITY OF THE SOLUTION OF A CERTAIN 


\section{ON THE PERIODICITY OF THE SOLUTION OF A CERTAIN NONLINEAR INTEGRAL EQUATION}

\section{Olavi Hellman}

In the following paper we will study the nonlinear integral equation

$$
E(t)=F(t)-\int_{0}^{t} G(t-\tau) N\{E(\tau)\} d \tau
$$

where $F(t)$ is a known periodic real function and $G(t)$ and $N(x)$ are known real functions. In particular we will investigate the behaviour of the solution $E(t)$ of the equation (1) for large values of $t$.

We assume that $G \in L[0, \infty]$ and that $N(x)$ is bounded almost everywhere and Borel-measurable in $[-\infty, \infty]$. Furthermore $N(x)$ is assumed expressible in the form

$$
N(x) \sim N(0)+\int_{-\infty}^{+\infty} S(\lambda) \frac{e^{i \lambda x}-1}{i \lambda} d \lambda
$$

with $\int_{-\infty}^{+\infty}|S(\lambda)| d \lambda<\infty$ and with finite $N(0)$. This representation is to be valid almost everywhere in $[-\infty, \infty]$

Because $N(x)$ is Borel-measurable in $[-\infty, \infty]$ and $|N(0)|<\infty$, the measurability of $x$ implies the measurability of $N(x)$. The following four classes of $N(x)$-functions are distinguished:

$$
\begin{array}{llll}
N \in K_{11} & \text { if } & x \in L[0,1] & \text { implies } N(x) \in L[0,1] \\
N \in K_{1 \infty} & \text { if } \quad x \in L[0,1] & \text { implies } N(x) \in L[0, \infty] \\
N \in K_{\infty 1} & \text { if } \quad x \in L[0, \infty] & \text { implies } N(x) \in L[0,1] \\
N \in K_{\infty \infty} & \text { if } \quad x \in L[0, \infty] & \text { implies } N(x) \in L[0, \infty]
\end{array}
$$

The space of measurable and bounded functions defined on the finite interval $[0, A]$ will be denoted by $M[0, A]$. The norm of $x \in M[0, A]$ is defined, as usual, by

$$
\|x\|=\inf _{E}\left\{\sup _{t \in[0, A]-E}|x|\right\}
$$

where $E$ ranges over the sets of measure zero in $[0, A]$, and the distance of $x \in M[0, A]$ and $y \in M[0, A]$ by $\|x-y\|$. The space $M[0,1]$ is complete.

The proofs in this paper will be based on the following theorem by Tihonov (see for instance [1]) which is valid in $M[0, A]$ : Let the operator $B$ map $M[0, A]$ into itself and let $\|B(x)-B(y)\| \leqq \beta\|x-y\|$ for all $x$ and

Received March 5, 1957. The preparation of this paper was sponsored by the Office of Naval Research and the Office of Ordnance Research, U.S. Army. Reproduction in whole or in part is permitted for any purpose of the U.S. Government. 
$y$ in $M[0, A]$, where $\beta<1$. Then the equation $y=B(y)$ has a unique solution $\bar{y}$ in $M[0, A]$. The function $\bar{y}$ may be obtained by iteration:

$$
\bar{y}=\lim _{n \rightarrow \infty} y_{n}
$$

where $y_{n}=B\left(y_{n-1}\right)$ and where $y_{0}$ may be taken arbitrarily from $M[0, A]$.

We will prove the following theorem.

THEOREM. Suppose that $F(t)$ is a periodic function in $[0, \infty]$ with period $T$, and that $F \in M[0, T]$. Furthermore suppose that $G \in L[0, \infty]$, $N \in K_{1 \infty}$ and

$$
\left(\int_{0}^{\infty}|G(u)| d u\right)\left(\int_{-\infty}^{+\infty}|S(\lambda)| d \lambda\right)<1 .
$$

If $E(t)$ is the solution of

$$
E(t)=F(t)-N(0) \int_{0}^{t} G(u) d u-\int_{0}^{t} G(t-\tau) \int_{-\infty}^{+\infty} S(\lambda) \frac{e^{i \lambda F(\tau)}-1}{i \lambda} d \lambda d \tau
$$

then $\lim E(n T+u)=v(u)$ exists, as $n \rightarrow \infty$ through integer values. The convergence is uniform. Moreover, $v(u)$ has the period $T$, and satisfies

$$
v(u)=F(u)-N(0) \int_{0}^{\infty} G(u) d u-\int_{0}^{\infty} G(\tau) \int_{-\infty}^{+\infty} S(\lambda) \frac{e^{i \lambda v(u-\tau)}-1}{i \lambda} d \lambda d \tau
$$

This equation can be solved by iteration stating with any element of $M[0, T]$. The solution of (5) is unique.

In order to prove the theorem, we will first prove two lemmas. Put

$$
H[\Delta(u+m T)]=\int_{0}^{t_{0}} G(\tau) d \tau \int_{-\infty}^{+\infty} S(\lambda) e^{i \lambda E(m T+u-\tau)} \frac{e^{i \lambda \Delta(m T+u-\tau)}-1}{i \lambda} d \lambda
$$

where $\Delta(u+m T)=E(u+n T)-E(u+m T)$ and $0 \leqq u \leqq T$. Here $T$ is a finite positive real number, $t_{0}$ a positive real number which may be finite or infinite and $m$ and $n$ positive integers. $E(u+n T) \in M[0, T]$ and $E(u+$ $m \mathrm{~T}) \in M[0, T]$ implies $\Delta(u+m T) \in M[0, T]$. The operator $H$ will play an important role in the following considerations. For this reason we will first establish some of its properties. We will write more briefly $H(\Delta(m T+u))=H(\Delta)$.

Lemma 1. Suppose that $G \in L[0, \infty]$, and suppose that the function $N(x)$ belongs to one of the classes $K_{11}$ and $K_{1 \infty}$. Then $\Delta \in M[0, T]$ implies $H(\Delta) \in M[0, T]$ and

$$
\left\|H\left(\Delta_{1}\right)-H\left(\Delta_{2}\right)\right\| \leqq \beta\left\|\Delta_{1}-\Delta_{2}\right\|
$$

where 


$$
\beta=\left(\int_{0}^{\infty}|G(u)| d u\right)\left(\int_{-\infty}^{+\infty}|S(\lambda)| d \lambda\right)
$$

Now

$$
\begin{aligned}
& \int_{0}^{t} G(\tau)\left[N\left\{E_{1}(t-\tau)\right\}-N\left\{E_{2}(t-\tau)\right\}\right] d \tau \\
= & \int_{0}^{t} G(\tau) I\left(t_{0}-\tau\right)\left[N\left\{E_{1}(t-\tau)\right\}-N\left\{\dot{E}_{2}(t-\tau)\right\}\right] d \tau
\end{aligned}
$$

where

$$
N(E)=\int_{-\infty}^{+\infty} S(\lambda) \frac{e^{i \lambda E}-1}{i \lambda} d \lambda
$$

and

$$
I\left(t_{0}-t\right)=\left\{\begin{array}{l}
1 \text { if } t \leqq t_{0} \\
0 \text { if } t_{0}<t
\end{array}\right.
$$

$G \in L[0, \infty]$ implies $G(\tau) I\left(t_{0}-\tau\right) \in \mathrm{L}[0, \infty]$. Furthermore, from $x \in M[0, T]$ and the properties of $N(x)$ follows that $N(x) \in M[0, T]$. Consequently $N(x) \in L[0, T]$. From known properties of the convolution follows now that

$$
\int_{0}^{t} G(\tau) I\left(t_{0}-\tau\right)\left[N\left\{E_{1}(t-\tau)\right\}-N\left\{E_{2}(t-\tau)\right\}\right] d \tau \in L[0, T] .
$$

Hence $H(\Delta) \in L[0, T]$. Now, as is easily seen,

$$
\|H(\Delta)\| \leqq\left(\int_{-\infty}^{+\infty}|S(\lambda)| d \lambda\right)\left\|\int_{0}^{t}|G(u)\|\Delta|(t+m T-u)| d u\| \leqq \beta\|\Delta\|\right.
$$

which implies the boundedness of $H(\Delta)$. The function $H(\Delta)$ is thus measurable and bounded in $[0, T], H(\Delta) \in M[0, T]$. Furthermore

$$
\begin{aligned}
\| H\left(\Delta_{2}\right) & -H\left(\Delta_{1}\right)\|=\| \int_{0}^{t_{0}} G(\tau) d \tau \int_{-\infty}^{+\infty} S(\lambda) e^{i \lambda E(u+m T-\tau)} \frac{e^{i \lambda \Delta_{2}(u+m T-\tau)}-e^{i \lambda \Delta_{1}(u+m T-\tau)}}{2} d \lambda \| \\
& \leqq\left(\int_{-\infty}^{+\infty}|S(\lambda)| d \lambda\right)\left\|\int _ { 0 } ^ { t _ { 0 } } \left|G(\tau)\left\|\Delta_{2}(u+m T-\tau)-\Delta_{1}(u+m T-\tau) \mid d \tau\right\|\right.\right. \\
& \leqq \beta\left\|\Delta_{2}-\Delta_{1}\right\|,
\end{aligned}
$$

which completes the proof.

We will now consider the norm

$$
\begin{gathered}
\| E(u+n T)-E(u+m T)+\int_{0}^{f(m)} G(\tau)[N\{E(u+n T-\tau)\} \\
-N\{E(u+m T-\tau)\}] d \tau \|=Q
\end{gathered}
$$

where $m$ and $n$ are positive integers, $f(m)$ an arbitrary function of $m$, 
$T$ a finite positive number and $E \in M[0, T]$. Furthermore it will be assumed that $G \in L[0, \infty]$ and $N \in K_{1 \infty}$ and that they satisfy the condition

$$
\left(\int_{-\infty}^{+\infty}|S(\lambda)| d \lambda\right)\left(\int_{0}^{\infty}|G(u)| d u\right)<1 \text {. }
$$

The following lemma holds.

LEMMA 2. For every $\varepsilon>0$ there exists an integer $m_{\mathrm{J}}$ such that $m \geqq m_{0}$ and $n \geqq m_{\mathrm{J}}$ imply $Q<\varepsilon$, if and only if, with $v(u)$ from $M[0, T]$, $\|E(u+p T)-v(u)\| \rightarrow 0$ as $p \rightarrow \infty$ through positive integral values.

Suppose first that $\|E(u+p T)-v(u)\| \rightarrow 0$, as $p \rightarrow \infty$, where $E$ and $v$ are in $M[0, T]$. Now

$$
\begin{aligned}
& \| \int_{0}^{f(m)} G(\tau)[N\{E(u+n T-\tau)\}-N\{E(u+m T-\tau)\}] d \tau \\
= & \left\|\int_{0}^{f(m)} G(\tau) \int_{-\infty}^{+\infty} S(\lambda) \frac{e^{i \lambda E(u+n T-\tau)}-e^{i \lambda E(u+m T-\tau)}}{i \lambda} d \lambda d \tau\right\| \\
\leqq & \left(\int_{0}^{\infty}|G(u)| d u\right)\left(\int_{-\infty}^{+\infty}|S(\lambda)| d \lambda\right)\|E(u+n T-\tau)-E(u+m T-\tau)\|
\end{aligned}
$$

and consequently

$$
\begin{aligned}
& \left\|E(u+n T)-E(u+m T)+\int_{0}^{f(m)} G(\tau)[N\{E(u+n T-\tau)\}-N\{E(u+m T-\tau)\}] d \tau\right\| \\
& \leqq\left[1+\left(\int_{0}^{\infty}|G(u)| d u\right)\left(\int_{-\infty}^{+\infty}|S(\lambda)| d \lambda\right)\right]\|E(u+n T)-E(u+m T)\|
\end{aligned}
$$

where $\left(\int_{0}^{\infty}|G(u)| d u\right)\left(\int_{-\infty}^{+\infty}|S(\lambda)| d \lambda\right)<1$. Because $\|E(u+p T)-v(u)\| \rightarrow 0$, as $p \rightarrow \infty$, there exists for every $\varepsilon>0$ an integer $m_{1}$ such that $m_{1} \leqq m<n$ implies

$$
\|E(u+n T)-E(u+m T)\| \leqq \frac{\varepsilon}{1+\beta}
$$

from which the first part of the lemma follows.

Suppose now that (6) is valid for $m$ and $n$ greater than a given integer $m_{2}$. The inequality (6) may be written

$$
\begin{aligned}
& \| \Delta(u+m T) \\
& \quad+\int_{0}^{f(m)} G(\tau) \int_{-\infty}^{+\infty} S(\lambda) e^{i \lambda E(u+m T-\tau)} \frac{e^{i \lambda \Delta(u+m T-\tau)}-1}{i \lambda} d \lambda d \tau \| \leqq \varepsilon
\end{aligned}
$$

where $\Delta(u+m T)=E(u+n T)-E(u+m T)$

Now let $h$ be a function in $M[0, T] \cap S(\varepsilon, 0)$ where $S(\varepsilon, 0)$ is the sphere with radius $\varepsilon$ and center at $h=0$. Put 


$$
\Delta(u+m T)
$$

$$
+\int_{0}^{f(m)} G(\tau) \int_{-\infty}^{+\infty} S(\lambda) e^{i \lambda E(u+m T-\tau)} \frac{e^{i \lambda \Delta(u+m T-\tau)}-1}{i \lambda} d \lambda d \tau=h(u)
$$

The functions $\Delta$ obtained by solving (8) for all $h \in M[0, T] \cap S(\varepsilon, 0)$ are those which satisfy (7). $E(u+m T)$ is a known function.

The equation

$$
\begin{aligned}
\Delta(u+m T) & =h(u)-\int_{0}^{f(m)} G(\tau) \int_{-\infty}^{+\infty} S(\lambda) e^{i \lambda E(u+m T-\tau)} \frac{e^{i \lambda \Delta(u+m T-\tau)}-1}{i \lambda} d \lambda d \tau \\
& =h(u)-H[\Delta(u+m T)]
\end{aligned}
$$

where $H$ is the operator defined on page 3, may be solved by iteration.

Indeed, by Lemma 1 the operator $H$ is defined in $M[0, T], \Delta \in M[0, T]$ implies $H(\Delta) \in M[0, T]$ and

$$
\left\|h(u)-H\left(\Delta_{1}\right)-\left(h(u)-H\left(\Delta_{2}\right)\right)\right\|=\left\|H\left(\Delta_{2}\right)-H\left(\Delta_{1}\right)\right\| \leqq \beta\left\|\Delta_{2}-\Delta_{1}\right\|
$$

where $\beta=\left(\int_{0}^{\infty}|G(u)| d u\right)\left(\int_{-\infty}^{+\infty}|S(\lambda)| d \lambda\right)<1$.

The conditions of the Tihonov's theorem are thus satisfied. We begin the iteration process with an $h$ from $M[0, T] \cap S(\varepsilon, 0)$ :

$$
\Delta_{1}(u+n T)=h(u)-\int_{0}^{f(m)} G(\tau) \int_{-\infty}^{+\infty} S(\lambda) e^{i \lambda E(u+n T-\tau)} \frac{e^{i \lambda h(u-\tau)}-1}{i \lambda} d \lambda d \tau
$$

and generally

$$
\Delta_{p+1}(u+n T)=h(u)-\int_{0}^{f(m)} G(\tau) \int_{-\infty}^{+\infty} S(\lambda) e^{i \lambda E(u+n T-\tau)} \frac{e^{i \lambda \Delta_{p}(u+m T-\tau)}-1}{i \lambda} d \lambda d \tau
$$

The unique solution of (9) is then $\lim _{k \rightarrow \infty} \Delta_{k}(u+n T)=\Delta(u+n T)$ where $\Delta(u+m T)$ is in $M[0, T]$.

Now

$$
\begin{aligned}
\left\|\Delta_{p+1}\right\| & \leqq h\|+\| \int_{0}^{f(m)}|G(\tau)| \int_{-\infty}^{+\infty}\left|S(\lambda)\left\|e^{i \lambda E(u+m T-\tau)}\right\| \frac{e^{i \lambda \Delta_{p}-1}}{i \lambda}\right| d \lambda d \tau|| \\
& \leqq \varepsilon+\left(\int_{0}^{\infty}|G(\lambda)| d u\right)\left(\int_{-\infty}^{+\infty}|S(\lambda)| d \lambda\right)\left\|\Delta_{p}\right\| \leqq \varepsilon+\beta\left\|\Delta_{p}\right\|
\end{aligned}
$$

From this inequality one obtains now, remembering that $\left\|\Delta_{0}\right\|=\|h\| \leqq \varepsilon$ and that $\beta<1$,

$$
\left\|\Delta_{p+1}\right\| \leqq\left(1+\beta+\beta^{2}+\cdots+\beta^{p+1}\right) \varepsilon \leqq \frac{\varepsilon}{1-\beta}
$$

This inequality holds true for all $p$. Consequently 


$$
\|\Delta(u+n T)\| \leqq \frac{\varepsilon}{1-\beta}
$$

or, in view of the definition of $\Delta(u+n T)$,

$$
\|E(u+n T)-E(u+m T)\| \leqq \frac{\varepsilon}{1-\beta}
$$

for $m$ and $n$ greater than $m_{2}$. But such $m_{2}$ exists for every $\varepsilon>0$. From this and from the completeness of the space $M[0, T]$ follows that there exists a $v_{1} \in M[0, T]$ such that

$$
\left\|E(u+p T)-v_{1}(u)\right\| \longrightarrow 0
$$

as $p \rightarrow \infty$ through integral values.

We now proceed to prove the Theorem.

Because of the periodicity of $F(t)$ one obtains from (1)

$$
\begin{aligned}
& E(u+n T)+\int_{0}^{u+n T} G(\tau) N\{E(u+n T-\tau)\} d \tau \\
& =E(u+m T)+\int_{0}^{u+m T} G(\tau) N\{E(u+m T-\tau)\} d \tau
\end{aligned}
$$

where $0 \leqq u \leqq T$ and where $m$ and $n$ are positive integers.

Suppose that $m<n$ and $t_{0} \leqq m T$. Then

$$
\begin{gathered}
E(u+n T)-E(u+m T)+\int_{0}^{t_{0}} G(\tau)[N\{E(u+n T-\tau)\}-N\{E(u+m T-\tau)\}] d \tau \\
=\int_{t_{0}}^{u+m T} G(\tau) N\{E(u+m T-\tau)\} d \tau-\int_{t_{0}}^{u+n T} G(\tau) N\{E(u+n T-\tau)\} d \tau
\end{gathered}
$$

and

$$
\begin{aligned}
& \left\|E(u+n T)-E(u+m T)+\int_{0}^{t_{0}} G(\tau)[N\{E(u+n T-\tau)\}-N\{E(u+m T-\tau)\}] d \tau\right\| \\
\leqq & \left\|\int_{t_{0}}^{u+n T}\left|G(\tau)\left\|N\{E(u+n T-\tau)\}\left|d \tau+\int_{t_{0}}^{u+m T}\right| G(\tau)\right\| N\{E(u+m T-\tau)\}\right| d \tau\right\| \\
\leqq & \left(\left\|\int_{t_{0}}^{u+n T}|G(\tau)| d \tau\right\|+\left\|\int_{t_{0}}^{u+m T}|G(\tau)| d \tau\right\|\right)\|N\| \leqq 2|| N \| \int_{t_{0}}^{\infty}|G(\tau)| d \tau
\end{aligned}
$$

Because $G \in L[0, \infty]$, there exists a positive integer $m_{3}$ for every $\varepsilon>0$ such that for $t_{\jmath}=m_{3} T$

$$
\int_{m_{3} T}^{\infty}|G(\lambda)| d u \leqq \frac{\varepsilon}{2\|N\|}
$$

But $m_{3} \leqq m<n$. Consequently, for every $\varepsilon>0$ there exists a positive integer $m_{3}$ such that $m_{3} \leqq m<n$ implies 


$$
\begin{aligned}
\| E(u+n T) & -E(u+m T) \\
& +\int_{0}^{m_{2} T} G(\tau)[N\{E(u+n T-\tau)\}-N\{E(u+m T-\tau)\}] d \tau \| \leqq \varepsilon
\end{aligned}
$$

By Lemma 2 it follows now that there exists a $v \in M[0, T]$ such that $\|E(u+p T)-v(u)\| \rightarrow 0$ as $p \rightarrow \infty$ through positive integral values. Consequently $E(u+p T)$ converges uniformly to $v(u)$ in $[0, T]$. That $v(u)$ is periodic with period $T$ is immediate.

We substitute now

$$
E(u+n T)=v(u)+H_{n}(u)
$$

where $H_{n} \in M[0, T]$ and $\left\|H_{n}\right\| \rightarrow 0$, as $n \rightarrow \infty$ and where $0 \leqq u \leqq T$, into (1) and obtain

$$
\begin{aligned}
v(u)+H_{n}(u)=F(u)-N(0) \int_{0}^{u+n T} G(\tau) d \tau \\
-\int_{0}^{u+n T+} G(\tau) \int_{-\infty}^{+\infty} S(\lambda) \frac{e^{i \lambda v(u-\tau)} e^{i \lambda H_{n}(u-\tau)}-1}{i \lambda} d \lambda d \tau
\end{aligned}
$$

As is seen at once, this may be rewritten as follows:

$$
\begin{aligned}
v(u) & -F(u)+\int_{0}^{\infty} G(\tau) \int_{-\infty}^{+\infty} S(\lambda) \frac{e^{i \lambda v(u-\tau)}-1}{i \lambda} d \lambda d \tau+N(0) \int_{0}^{\infty} G(\tau) d \tau \\
& +\int_{0}^{\infty} G(\tau) \int_{-\infty}^{+\infty} S(\lambda) e^{i \lambda v(u-\tau)} \frac{e^{i \lambda H_{n}(u-\tau)}-1}{i \lambda} d \lambda d \tau+H_{n}(u)+ \\
& -\int_{n T+u}^{\infty} G(\tau) \int_{-\infty}^{+\infty} S(\lambda) \frac{e^{i \lambda v(u-\tau)} e^{i \lambda H_{n}(u-\tau)}-1}{i \lambda} d \lambda d \tau-N(0) \int_{n T+u}^{\infty} G(\tau) d \tau=0
\end{aligned}
$$

which yields the inequality

$$
\begin{aligned}
& \| v(u)- F(u)+\int_{0}^{\infty} G(\tau) \int_{-\infty}^{+\infty} S(\lambda) \frac{e^{i \lambda v(u-\tau)}-1}{i \lambda} d \lambda d \tau \| \\
& \leqq \\
& \quad\left\|H_{n}(u)\right\|+\left(\int_{0}^{\infty}|G(u)| d u\right)\left(\int_{-\infty}^{+\infty}|S(\lambda)| d \lambda\right)\left\|H_{n}(u)\right\| \\
& \quad+\left(\int_{n T+u}^{\infty}|G(u)| d u\right)\left(\int_{-\infty}^{+\infty}|S(\lambda)| d \lambda\right)|| v(u)+H_{n}(u) \|+N(0) \int_{n T+u}^{\infty}|G(u)| d u \\
&=(1+\beta)\left\|H_{n}(u)\right\|+\left(\int_{n T}^{\infty}|G(u)| d u\right)\left(\int_{-\infty}^{+\infty}|S(\lambda)| d \lambda\right)\left(\|v(u)\|+\left\|H_{n}(u)\right\|\right) \\
& \quad+N(0) \int_{n T}^{\infty}|G(u)| d \lambda .
\end{aligned}
$$

But $\beta, \int_{-\infty}^{+\infty}|S(\lambda)| d \lambda,\|v(u)\|$ and $N(0)$ are finite, $\left\|H_{n}\right\| \rightarrow 0$ as $n \rightarrow \infty$ and $\int_{n T}^{\infty}|G(u)| d u \rightarrow 0$ as $n \rightarrow \infty$. Consequently 


$$
\left\|v(u)-\left(F(u)-N(0) \int_{0}^{\infty} G(u) d u-\int_{0}^{\infty} G(\tau) \int_{-\infty}^{+\infty} S(\omega) \frac{e^{i \lambda v(u-\tau)}-1}{i \lambda} d \lambda d \tau\right)\right\| \longrightarrow 0,
$$

as $n \rightarrow \infty$ through integral values, from which the equation (5) follows for $v(u)$.

The right side of (5) satisfies the conditions of Tihonov's theorem. This follows by Lemma 1 where we substitute $t_{0}=\infty, E(m T+u-\tau)=0$ and $\Delta(m T+u-\tau)=v(u-\tau)$. If the right side of (5) is denoted by $c(v)$, then, by Lemma $1, v \in M[0, T]$ implies $c(v) \in M[0, T]$ and $\left\|c\left(v_{1}\right)-c\left(v_{2}\right)\right\|$ $\leqq \beta\left\|v_{1}-v_{2}\right\|$ for $v_{1}$ and $v_{2}$ from $M[0, T]$. By Tihonov's theorem it follows then that the equation (5) has a unique solution $v \in M[0, T]$ which may be obtained by iteration, beginning with an arbitrary function from $M[0], T$.

\section{REFERENCE}

1. Г. Элсголч, Качественная теория бифферентиалных уравнениях. Гостехиздат 1955, MOCKGA

UNIVERSity OF CALIFuRNIA AT LOS ANGELES 


\section{PACIFIC JOURNAL OF MATHEMATICS}

\section{EDITORS}

\section{H. L. Royden}

Stanford University

Stanford, California

\section{R. A. Beaumont}

University of Washington

Seattle 5 , Washington

\author{
A. L. Whiteman
}

University of Southern California

Los Angeles 7, California

E. G. Straus

University of California

Los Angeles 24, California

\section{ASSOCIATE EDITORS}
E. F. BECKENBACH
A. HORN
L. NACHBIN
G. SZEKERES
C. E. BURGESS
V. GANAPATHY IYER
I. NIVEN
F. WOLF
M. HALL
R. D. JAMES
T. G. OSTROM
E. HEWITT
M. S. KNEBELMAN
M. M. SCHIFFER
K. YOSIDA

\section{SUPPORTING INSTITUTIONS}

\author{
UNIVERSITY OF BRITISH COLUMBIA \\ CALIFORNIA INSTITUTE OF TECHNOLOGY \\ UNIVERSITY OF CALIFORNIA \\ MONTANA STATE UNIVERSITY \\ UNIVERSITY OF NEVADA \\ OREGON STATE COLLEGE \\ UNIVERSITY OF OREGON \\ UNIVERSITY OF SOUTHERN CALIFORNIA
}

\author{
STANFORD UNIVERSITY \\ UNIVERSITY OF UTAH \\ WASHINGTON STATE COLLEGE \\ UNIVERSITY OF WASHINGTON \\ * * * * \\ AMERICAN MATHEMATICAL SOCIETY \\ CALIFORNIA RESEARCH CORPORATION \\ HUGHES AIRCRAFT COMPANY \\ THE RAMO-WOOLDRIDGE CORPORATION
}

Mathematical papers intended for publication in the Pacific Journal of Mathematics should be typewritten (double spaced), and the author should keep a complete copy. Manuscripts may be sent to any of the editors. All other communications to the editors should be addressed to the managing editor, E. G. Straus at the University of California, Los Angeles 24, California.

50 reprints per author of each article are furnished free of charge; additional copies may be obtained at cost in multiples of 50 .

The Pacific Journal of Mathematics is published quarterly, in March, June, September, and December. The price per volume (4 numbers) is $\$ 12.00$; single issues, $\$ 3.50$. Back numbers are available. Special price to individual faculty members of supporting institutions and to individual members of the American Mathematical Society: $\$ 4.00$ per volume; single issues, $\$ 1.25$.

Subscriptions, orders for back numbers, and changes of address should be sent to Pacific Journal of Mathematics, 2120 Oxford Street, Berkeley 4, California.

Printed at Kokusai Bunken Insatsusha (International Academic Printing Co., I.td.), No. 10, 1-chome, Fujimi-cho, Chiyoda-ku, Tokyo, Japan.

PUBLISHED BY PACIFIC JOURNAL OF MATHEMATICS, A NON-PROFIT CORPORATION

The Supporting Institutions listed above contribute to the cost of publication of this Journal, but they are not owners or publishers and have no responsibility for its content or policies. 


\section{Pacific Journal of Mathematics}

\section{Vol. 8, No. 2 \\ April, 1958}

John Herbert Barrett, Second order complex differential equations with a real independent variable ............................ 187

Avner Friedman, Remarks on the maximum principle for parabolic equations and its applications ......................... 201

Richard Robinson Goldberg, An inversion of the Stieltjes transform ....... 213

Olavi Hellman, On the periodicity of the solution of a certain nonlinear integral equation .................................. 219

Gilbert Helmberg, A theorem on equidistribution on compact groups...... 227

Lloyd Kenneth Jackson, Subfunctions and the Dirichlet problem ......... 243

Naoki Kimura, The structure of idempotent semigroups. I ............ 257

Stephen Kulik, A method of approximating the complex roots of equations........................................ 277

Ancel Clyde Mewborn, A note on a paper of L. Guttman.............. 283

Zeev Nehari, On the principal frequency of a membrane ............ 285

G. Pólya and I. J. Schoenberg, Remarks on de la Vallée Poussin means and convex conformal maps of the circle ...................... 295

B. M. Stewart, Asymmetry of a plane convex set with respect to its centroid .......................................... 335

Hans F. Weinberger, Lower bounds for higher eigenvalues by finite difference methods

Edwin Weiss and Neal Zierler, Locally compact division rings ......... 369

Bertram Yood, Homomorphisms on normed algebras ................. 373 